\title{
On the Turán number of forests
}

\author{
Bernard Lidický Hong Liu Cory Palmer* \\ Department of Mathematical Sciences, \\ University of Illinois at Urbana-Champaign, \\ Urbana, Illinois 61801, USA \\ \{lidicky,hliu, ctpalmer\}@illinois.edu
}

Submitted: Feb 18, 2013; Accepted: Jun 21, 2013; Published: Jun 30, 2013

Mathematics Subject Classifications: 05C05, 05C35

\begin{abstract}
The Turán number of a graph $H$, ex $(n, H)$, is the maximum number of edges in a graph on $n$ vertices which does not have $H$ as a subgraph. We determine the Turán number and find the unique extremal graph for forests consisting of paths when $n$ is sufficiently large. This generalizes a result of Bushaw and Kettle [Combinatorics, Probability and Computing 20:837-853, 2011]. We also determine the Turán number and extremal graphs for forests consisting of stars of arbitrary order.
\end{abstract}

\section{Introduction}

Notation in this paper is standard. For a graph $G$ let $E(G)$ be the set of edges and $V(G)$ be the set of vertices. The order of a graph is the number of vertices. The number of edges of $G$ is denoted by $e(G)=|E(G)|$. For a graph $G$ with subgraph $H$, the graph $G-H$ is the induced subgraph on vertex set $V(G) \backslash V(H)$ i.e. $G[V(G) \backslash V(H)]$. For $U \subset V(G)$ we define $N(U)$ to be the set of vertices in $V(G) \backslash U$ that have a neighbor in $U$, while the common neighborhood of $U \subset V(G)$ is the set of vertices in $V(G) \backslash U$ that are adjacent to every vertex in $U$. We denote the degree of a vertex $v$ by $d(v)$ and the minimum degree in a graph by $\delta(G)$. A universal vertex in $G$ is a vertex that is adjacent to all other vertices in $G$. A star forest is a forest whose connected components are stars and a linear forest is a forest whose connected components are paths. A path on $k$ vertices is denoted $P_{k}$ and a star with $k+1$ vertices is denoted $S_{k}$. Superscript is used to denote the index of a particular graph in a set of graphs. Let $k \cdot H$ denote the graph of the disjoint union of $k$ copies of the graph $H$.

${ }^{*}$ Research partially supported by OTKA NK 78439 
The Turán number, ex $(n, H)$, of a graph $H$ is the maximum number of edges in a graph on $n$ vertices which does not contain $H$ as a subgraph. The problem of determining Turán numbers is one of the cornerstones of graph theory. A traditional starting point of extremal graph theory is a theorem of Mantel (see e.g. [1]) that the maximum number of edges in a triangle-free graph on $n$ vertices is $\left\lfloor\frac{n^{2}}{4}\right\rfloor$. Turán $[17,18]$ generalized this result to find the extremal graph of any complete graph. In particular he showed that $\operatorname{ex}\left(n, K_{r}\right)=\left(\frac{r-2}{r-1}\right) \frac{n^{2}}{2}$. The Erdős-Stone theorem [9] states that asymptotically Turán's construction is best-possible for any $r$-chromatic graph $H$ (as long as $r>2$ ). More precisely ex $(n, H)=\left(\frac{r-2}{r-1}\right) \frac{n^{2}}{2}+o\left(n^{2}\right)$. Thus when $H$ is bipartite Erdös-Stone only states that $\operatorname{ex}(n, H)=o\left(n^{2}\right)$. There are many classical theorems on determining the Turán number of bipartite graphs. For example for complete bipartite graphs the Kövári-SósTurán theorem [15] gives $\operatorname{ex}\left(n, K_{s, t}\right)=O\left(n^{2-1 / s}\right)$ (also see e.g. [8, 4, 11]) and for even cycles Bondy and Simonovits [2] have ex $\left(n, C_{2 k}\right) \leqslant n^{1+1 / k}$.

In 1959, Erdős and Gallai [7] determined the Turán number for paths. We state the theorem here since it is an important tool in our proofs.

Theorem 1 ([7]). For any $k, n>1, \operatorname{ex}\left(n, P_{k}\right) \leqslant \frac{k-2}{2} n$, where equality holds for the graph of disjoint copies of $K_{k-1}$.

A well-known conjecture of Erdős and Sós [6] states that the Turán number for paths is enough for any tree i.e. a graph $G$ on $n$ vertices and more than $\frac{k-2}{2} n$ edges contains any tree on $k$ vertices. A proof of the Erdős-Sós conjecture for large trees was announced by Ajtai, Komlós, Simonovits and Szemerédi.

A natural extension of the problem is the determination of the Turán number of forests. Erdős and Gallai [7] considered the graph $H$ consisting of $k$ independent edges (note that $H$ is a linear forest) and found $\operatorname{ex}(n, H)=\max \left\{\left(\begin{array}{c}k-1 \\ 2\end{array}\right)+(k-1)(n-k+\right.$ $\left.1),\left(\begin{array}{c}2 k-1 \\ k\end{array}\right)\right\}$. When $n$ is large enough compared to $k$, the first bound in the maximum is larger, and the extremal graph attaining this bound is obtained by adding $k-1$ universal vertices to an independent set of $n-k+1$ vertices. This construction clearly does not contain $k$ independent edges as every such edge must include at least one of the universal vertices. This construction forms a model for the constructions presented throughout the paper. Brandt [3] generalized the above result by proving that any graph $G$ with $e(G)>\max \left\{\left(\begin{array}{c}k-1 \\ 2\end{array}\right)+(k-1)(n-k+1),\left(\begin{array}{c}2 k-1 \\ k\end{array}\right)\right\}$ contains every forest on $k$ edges without isolated vertices. An analogue of Erdős-Gallai theorem for linear paths in hypergraphs was considered by Füredi, Jiang and Seiver [12].

Recently, Bushaw and Kettle [5] found (among other things) the Turán number and extremal graph for linear forests where all components have even order as well as for linear forests where all components have the same odd order.

Motivated by these results we determined the Turán number and extremal graph for arbitrary linear forests. 
Theorem 2. Let $F$ be a linear forest with components of order $v_{1}, v_{2}, \ldots, v_{k}$. If at least one $v_{i}$ is not 3 , then for $n$ sufficiently large,

$$
\operatorname{ex}(n, F)=\left(\sum_{i=1}^{k}\left\lfloor\frac{v_{i}}{2}\right\rfloor-1\right)\left(n-\sum_{i=1}^{k}\left\lfloor\frac{v_{i}}{2}\right\rfloor+1\right)+\left(\begin{array}{c}
\sum_{i=1}^{k}\left\lfloor\frac{v_{i}}{2}\right\rfloor-1 \\
2
\end{array}\right)+c,
$$

where $c=1$ if all $v_{i}$ are odd and $c=0$ otherwise. Moreover, the extremal graph is unique.

Notice that the theorem avoids the case of linear forest with every component of order three. This case was solved by Bushaw and Kettle [5] and settled a conjecture of Gorgol [14]. We prove Theorem 2 in Section 2 and describe the unique $F$-free graph on $n$ vertices with ex $(n, F)$ edges.

Another motivation is the following conjecture of Goldberg and Magdon-Ismail [13]:

Let $F$ be a forest with $k$ components, then every graph $G$ with at least $e(F)+k$

vertices and average degree $>e(F)-1$ contains $F$ as a subgraph.

This is a natural generalization of the Erdös-Sós conjecture, however it is false. From Theorem 2, some simple calculation shows that the conjecture fails for linear forests with at least two components of even order.

We also investigate the other extreme case when each component is a star and we determine the Turán number and also find all the extremal graphs.

Theorem 3. Let $F=\bigcup_{i=1}^{k} S^{i}$ be a star forest where $d_{i}$ is the maximum degree of $S^{i}$ and $d_{1} \geqslant d_{2} \geqslant \cdots \geqslant d_{k}$. For $n$ sufficiently large,

$$
\operatorname{ex}(n, F)=\max _{1 \leqslant i \leqslant k}\left\{(i-1)(n-i+1)+\left(\begin{array}{c}
i-1 \\
2
\end{array}\right)+\left\lfloor\frac{d_{i}-1}{2}(n-i+1)\right\rfloor\right\} .
$$

Note that for a single component, Theorem 3 describes what is technically the Turán number of a star i.e. the maximum number of edges in a graph of fixed maximum degree. Furthermore, Theorem 3 includes the forest where every component is of order 3 (proved in [5]). In other words a forest of components each of order 3 behaves like a star forest rather than a linear forest. We prove Theorem 3 and characterize all extremal graphs in Section 3. In the proofs of Theorems 2 and 3 we make no attempt to minimize the bound on $n$. The last section contains a result about forests with small components.

\section{Linear forests}

We first consider the Turán problem for a linear forest. Throughout this section, unless otherwise specified, $F$ is a linear forest i.e. $F=\bigcup_{i=1}^{k} P^{i}$, such that $P^{i}$ is a path on $v_{i}$ vertices and $v_{1} \geqslant v_{2} \geqslant \ldots \geqslant v_{k} \geqslant 2$.

Let $F$ be a linear forest where at least one $v_{i}$ is not equal to 3 . Define $G_{F}(n)$ to be the graph on $n$ vertices with a set, $U$, of $\left(\sum_{i=1}^{k}\left\lfloor v_{i} / 2\right\rfloor\right)-1$ universal vertices together with a single edge in $G_{F}(n)-U$ if each $v_{i}$ is odd or $n-|U|$ independent vertices otherwise 


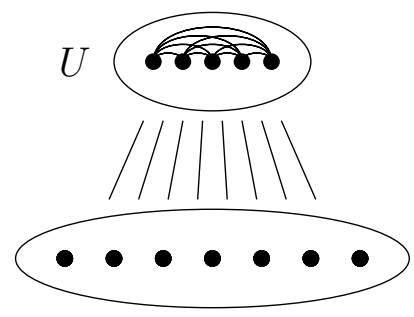

(a)

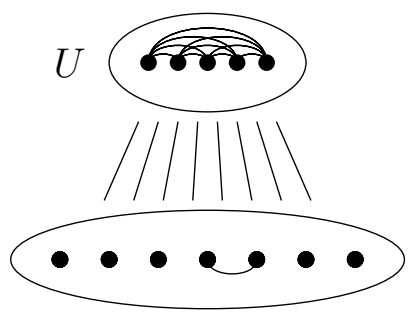

(b)

Figure 1: $G_{F}(n),(\mathrm{a})$ is the case where at least one path $F$ is of even order, (b) is the case where all paths in $F$ are of odd order.

(see Figure 1). Observe that $G_{F}(n)$ is $F$-free. Indeed, any path $P^{i}$ in $G_{F}(n)$ must use at least $\left\lfloor v_{i} / 2\right\rfloor$ vertices from $U$ but $|U|<\sum_{i=1}^{k}\left\lfloor v_{i} / 2\right\rfloor$. We show that $G_{F}(n)$ is the unique extremal graph for the linear forest $F$. We begin with a lemma that is a special case of Lemma 2.3 in [5]. We include a proof for the sake of completeness.

Lemma 4. Let $p, v \geqslant 2$ be given and let $n$ be at least $4 p(p+v)\left(\begin{array}{c}p \\ |p / 2|\end{array}\right)$. If $G$ is a graph on $n$ vertices with a set $P$ of $p$ vertices such that $e(G)-e(G-P)-\left(\begin{array}{l}p \\ 2\end{array}\right) \geqslant\left(\lfloor p / 2\rfloor-\frac{1}{2}\right) n$, then $P$ contains a subset of $\lfloor p / 2\rfloor$ vertices with a common neighborhood of $v+p$ vertices in $G-P$.

Proof. Let $n_{0}$ be the number of vertices in $G-P$ with at least $\lfloor p / 2\rfloor$ neighbors in $P$. Therefore the number of edges between $P$ and $G-P$ is at most $n_{0} p+\left(n-p-n_{0}\right)(\lfloor p / 2\rfloor-1)$. So

$$
e(G)-e(G-P)-\left(\begin{array}{l}
p \\
2
\end{array}\right) \leqslant n_{0} p+\left(n-p-n_{0}\right)(\lfloor p / 2\rfloor-1) .
$$

Rearranging terms gives $n_{0} \geqslant \frac{n / 2}{\lceil p / 2\rceil+1}$. There are $\left(\begin{array}{c}p \\ \lfloor p / 2\rfloor\end{array}\right)$ sets of $\lfloor p / 2\rfloor$ vertices in $P$. Thus there is some set with a common neighborhood of order at least

$$
\frac{n_{0}}{\left(\begin{array}{c}
p \\
\lfloor p / 2\rfloor
\end{array}\right)} \geqslant v+p
$$

First, let us consider the base case when $F$ consists of only two paths. The case when the two paths have the same order is resolved in [5], so let us assume they have different order.

Theorem 5. Suppose $F=P_{a} \cup P_{b}$, with $a>b \geqslant 2$. Let $G$ be any $F$-free $n$-vertex graph with $n \geqslant 8 a^{2}(a+b)^{2}\left(\begin{array}{c}a \\ \lfloor a / 2\rfloor\end{array}\right)^{2}$. Then $e(G) \leqslant e\left(G_{F}(n)\right)$, with equality only when $G \simeq G_{F}(n)$.

We use a standard trick to reduce the problem to graphs with large minimum degree. 
Lemma 6. Suppose $F=P_{a} \cup P_{b}$, with $a>b \geqslant 2$. Let $G$ be any $F$-free $n$-vertex graph with $n \geqslant 4 a(a+b)\left(\begin{array}{c}a \\ \lfloor a / 2\rfloor\end{array}\right)$ and $\delta(G) \geqslant\lfloor a / 2\rfloor+\lfloor b / 2\rfloor-1$. Then $e(G) \leqslant e\left(G_{F}(n)\right)$, with equality only when $G \simeq G_{F}(n)$.

We first show how Lemma 6 implies Theorem 5 and we give the proof of the lemma afterwards.

Proof of Theorem 5 using Lemma 6. Suppose $G$ is an extremal graph for $F$ on $n \geqslant 8 a^{2}(a+b)^{2}\left(\begin{array}{c}a \\ \lfloor a / 2\rfloor\end{array}\right)^{2}$ vertices and $e(G) \geqslant e\left(G_{F}(n)\right)$. We start by removing vertices of small degree from $G$. Suppose that there exists a vertex $v$ in $G$ with $d(v)<\delta\left(G_{F}(n)\right)=$ $\lfloor a / 2\rfloor+\lfloor b / 2\rfloor-1$. Let $G^{n}=G$. We then define $G^{n-1}=G^{n}-\{v\}$. We construct each successive $G^{i-1}$ from $G^{i}$ by removing a vertex of degree less than $\delta\left(G_{F}(i)\right)$. The process continues while $\delta\left(G^{i}\right)<\delta\left(G_{F}(i)\right)$. Notice that

$$
e\left(G^{i-1}\right)-e\left(G_{F}(i-1)\right) \geqslant e\left(G^{i}\right)-e\left(G_{F}(i)\right)+1 .
$$

Hence the process terminates after $n-\ell$ steps. We get $G^{\ell}$ with $\delta\left(G^{\ell}\right) \geqslant \delta\left(G_{F}(\ell)\right)$ and

$$
\left(\begin{array}{l}
\ell \\
2
\end{array}\right) \geqslant e\left(G^{\ell}\right) \geqslant e\left(G_{F}(\ell)\right)+n-\ell=(\lfloor a / 2\rfloor+\lfloor b / 2\rfloor-1) \ell+n-\ell+O\left(a^{2} b^{2}\right) \geqslant n,
$$

this implies $\ell>\sqrt{2 n} \geqslant 4 a(a+b)\left(\begin{array}{c}a \\ \lfloor a / 2\rfloor\end{array}\right)$. Since $e\left(G^{\ell}\right)>e\left(G_{F}(\ell)\right)$, Lemma 6 then implies $F \subseteq G^{\ell} \subseteq G$, a contradiction.

We give two useful lemmas before proving Lemma 6 . Let $H$ be a graph and let $U \subset V(H)$ such that $U$ has at least $|U|+3$ common neighbors in $V(H)-U$. Furthermore, let $1 \leqslant c \leqslant|U|+1$.

Lemma 7. Let $v \in N(U)$ be an endpoint of $P_{t}$ in $H-U$ where $1 \leqslant t \leqslant 3$. Then $H$ contains a path $P$ of order $2 c-2+t$ such that $|P \cap U|=c-1$.

Proof. Let $u \in U$ be a neighbor of $v$. Let $P^{\prime}$ be a path in $H$ of order $2 c-2$ starting at $u$ and avoiding vertices of $P_{t}$ and having at most $c-1$ vertices of $U$. It can be obtained from $U$ and $N(U) \backslash V\left(P_{t}\right)$ by alternating between $U$ and $N(U) \backslash V\left(P_{t}\right)$ since $U$ has at least $|U|+3$ common neighbors and $|U|+3 \geqslant|U|+t$. The path $P_{t}$ can be used to extend $P^{\prime}$ and finding a path of order $2 c-2+t$. See Figure 2(a) for case $t=3$.

Lemma 8. If there exist nonadjacent $u, v \in N(U)$ both of degree at least one in $H-U$ then $H$ contains a path $P$ of order $2 c+1$ such that $|P \cap U|=c-1$.

Proof. If there is a common neighbor of $u$ and $v$, then the claim follows from Lemma 7 because $u$ is an endpoint of a path of order three. Let $u^{\prime}$ and $v^{\prime}$ be distinct neighbors of $u$ and $v$, respectively, in $H-U$. A greedily obtainable path with end vertices $u$ and $v$ avoiding $u^{\prime}$ and $v^{\prime}$ of order $2 c-1$ can be extended by $u^{\prime}$ and $v^{\prime}$ to a path of order $2 c+1$. See Figure 2(b). 


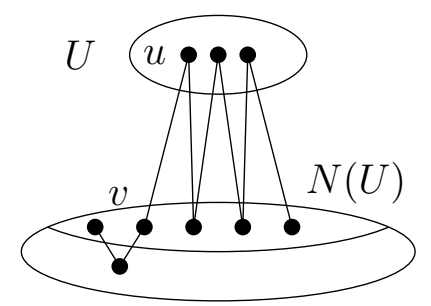

(a)

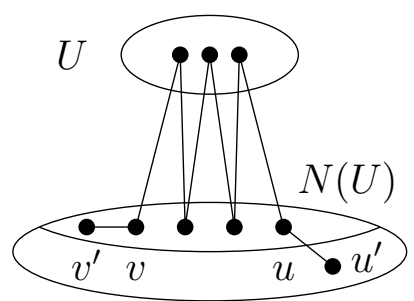

(b)

Figure 2: Finding a long path.

Proof of Lemma 6 . Let $G$ be an extremal graph for $F$ with $\delta(G) \geqslant\lfloor a / 2\rfloor+\lfloor b / 2\rfloor-1$. First we show that there is a set $U_{a}$ of $\lfloor a / 2\rfloor$ vertices with a common neighborhood of more than $a+b$ vertices. Such a set $U_{a}$ can be easily extended to a copy of $P_{a}$, which implies $G-U_{a}$ must be $P_{b}$-free. However, $G-U_{a}$ is not $P_{b-2}$-free and hence we can find a set $U_{b}$ of $\lfloor b / 2\rfloor-1$ vertices that share a large common neighborhood. Then we show that $G-U_{a}-U_{b}$ has at most one edge, which then implies $G \subseteq G_{F}(n)$.

Because $e(G) \geqslant e\left(G_{F}(n)\right)>\operatorname{ex}\left(n, P_{a}\right)$, we have $P_{b} \subseteq P_{a} \subseteq G$. The graph $G$ does not contain the forest $P_{a} \cup P_{b}$, so the graph $G-P_{a}$ is $P_{b}$-free. Thus $e\left(G-P_{a}\right) \leqslant \frac{b-2}{2}(n-a)$ by Theorem 1 (Erdős-Gallai). Because $e(G) \geqslant e\left(G_{F}(n)\right)$, the number of edges between $P_{a}$ and $G-P_{a}$ is at least $e\left(G_{F}(n)\right)-\frac{b-2}{2}(n-a)-\left(\begin{array}{l}a \\ 2\end{array}\right)$. Thus, by Lemma 4 , there is a set $U_{a} \subset P_{a}$ such that $\left|U_{a}\right|=\lfloor a / 2\rfloor$ and $U_{a}$ has at least $a+b$ many common neighbors.

Now observe that $G-U_{a}$ is $P_{b}$-free. Indeed, suppose there is a copy of $P_{b}$ in $G-U_{a}$, then $U_{a}$ can be extended to $P_{a}$ avoiding vertices of $P_{b}$, since $U_{a}$ has more than $a+b$ common neighbors. Thus $F \subseteq G$ which is a contradiction.

We now distinguish three cases based on the value of $b$. If $b=2$, then $G-U_{a}$ is $P_{2}$-free, namely $G-U_{a}$ is an independent set. Thus $G \subseteq G_{F}(n)$.

If $b=3$, then $G-U_{a}$ is $P_{3}$-free. Thus $G-U$ contains only isolated edges and vertices. If $u v$ is an isolated edge in $G-U_{a}$ then both $u$ and $v$ have a neighbor in $U_{a}$ as the minimum degree of $G$ is $\delta(G) \geqslant\lfloor a / 2\rfloor \geqslant 2$. Furthermore, at most one of $u$ and $v$ is not adjacent to all vertices of $U_{a}$, otherwise the graph $G_{0}$ obtained from $G$ by removing the edge $u v$ and adding all edges between $\{u, v\}$ and $U_{a}$ has more edges and remains $F$-free, which contradicts the extremality of $G$. The graph $G_{0}$ is indeed $F$-free. If not, then a copy of $F$ in $G_{0}$ contains at least one of $u, v$. However, $u$ and $v$ are isolated vertices in $G_{0}-U_{a}$. Hence they could be replaced by some of the $a+b$ common neighbors of $U_{a}$, which would give a copy of $F$ in $G$, a contradiction. Let $z$ be a vertex in $U_{a}$. If $a$ is even, then $U_{a} \backslash z$ together with an edge in $G-U_{a}$ complete a copy of $P_{a}$, and $N(z) \cup\{z\}$ contains a $P_{3}=P_{b}$ (see Figure 3(a)), thus $F \subseteq G$, contradiction. So $G-U_{a}$ is an independent set if $a$ is even. When $a$ is odd, then two edges in $G-U_{a}$ together with $U_{a} \backslash z$ completes a copy of $P_{a}$, and again $N(z) \cup\{z\}$ contains a $P_{b}$ (see Figure $3(\mathrm{~b})$ ), thus $G-U_{a}$ has at most one edge. Therefore, $G \subseteq G_{F}(n)$.

Now we may assume $b \geqslant 4$. Let $\left|V\left(G-U_{a}\right)\right|=n^{\prime}$. Denote by $G^{\prime}$ the graph obtained 


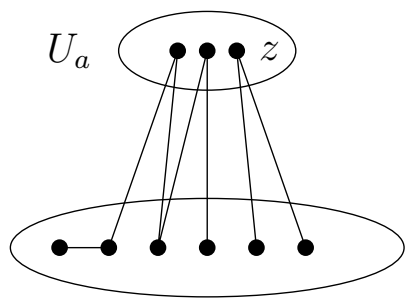

(a)

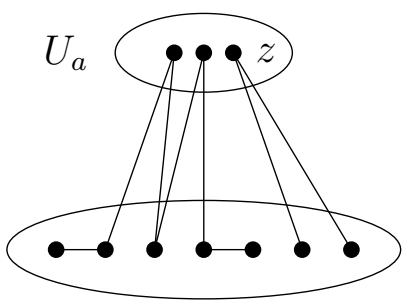

(b)

Figure 3: Cases for $b=3$.

by deleting $\lfloor a / 2\rfloor$ universal vertices from $G_{F}(n)$, clearly $\left|V\left(G^{\prime}\right)\right|=n^{\prime}$.

If $G-U_{a}$ is $P_{b-2}$-free, then $e\left(G-U_{a}\right) \leqslant\left(\frac{b}{2}-2\right) n^{\prime}<\left(\left\lfloor\frac{b}{2}\right\rfloor-1\right) n^{\prime}-\left(\begin{array}{c}\left\lfloor\frac{b}{2}\right\rfloor \\ 2\end{array}\right)=e\left(G^{\prime}\right)$, implying $e(G)<e\left(G_{F}(n)\right)$ which is a contradiction. Thus we may assume $G-U_{a}$ is not $P_{b-2}$-free. Take a maximum path, $P$, in $G-U_{a}$ and let $u$ be an end vertex of $P$. Since $G-U_{a}$ is $P_{b}$-free, $P$ has at most $b-1$ vertices. Then

$d(u)-(|V(P)|-1) \geqslant \delta(G)-b+2 \geqslant\lfloor a / 2\rfloor+\lfloor b / 2\rfloor-1-b+2=\lfloor a / 2\rfloor-\lceil b / 2\rceil+1 \geqslant 1$.

Thus $u$ has a neighbor, say $w$, not in $P$. Furthermore $w$ must be in $U_{a}$, since otherwise we could extend $P$ to a longer path in $G-U_{a}$. Recall that $U_{a}$ has more than $a+b$ common neighbors. Then $P$ with $w$ and a neighbor of $w$ in $G-U_{a}-P$ form a $P_{b}$. By Lemma 4 there is a set of $\lfloor b / 2\rfloor$ vertices of $P_{b}$ with a common neighborhood of order at least $a+b$ (by the same argument as before). Note that all vertices in this $P_{b}$ except $w$ are in $G-U_{a}$. Thus we find a set of vertices in $G-U_{a}$ of order $\lfloor b / 2\rfloor-1$, call it $U_{b}$, with a large common neighborhood $\left(U_{b}\right.$ is nonempty since $\left.b \geqslant 4\right)$. We are done if we show $G-U_{a}-U_{b}$ is empty if one of $a$ and $b$ is even or has at most one edge when they are both odd, as this implies $G \subseteq G_{F}(n)$.

Suppose that $G$ is not connected. Then there exists a connected component $C$ in $G$ not containing $U_{a}$. The minimum degree $\lfloor a / 2\rfloor+\lfloor b / 2\rfloor-1 \geqslant b-1$ implies that it is possible to find $P_{b}$ in $C$ (for example by a greedy algorithm). It contradicts the claim that $G-U_{a}$ is $P_{b}$-free. Hence we assume that $G$ is connected.

Notice that if a vertex is not adjacent to all vertices of $U_{a} \cup U_{b}$ then it has degree at least 1 in $G-U_{a}-U_{b}$ because of the minimum degree condition. If there are at least three such vertices, Lemma 7 or Lemma 8 applies with $H=G-U_{a}, U=U_{b}$ and $c=\left\lfloor\frac{b}{2}\right\rfloor$. It leads to the existence of a path of order $2\left\lfloor\frac{b}{2}\right\rfloor+1$ in $G-U_{a}$ which is a contradiction. Hence there are at most two vertices in $N\left(U_{b}\right) \backslash U_{a}$ that are not adjacent to all vertices of $U_{a}$.

Put $U=U_{a} \cup U_{b}$. The previous observation together with the fact that vertices of $U_{b}$ have many common neighbors implies that there is a common neighborhood of $U$ of order at least $\frac{n}{b\left(\begin{array}{c}b \\ b / 2\end{array}\right)}-2$. Hence whenever we find in $G$ a path of order $a$ using at most $\left\lfloor\frac{a}{2}\right\rfloor-1$ vertices of $U$ or a path of order $b$ using at most $\left\lfloor\frac{b}{2}\right\rfloor-1$ vertices of $U$ then we can easily find the other path of $F$. 
If a vertex $v$ in $G-U$ is not adjacent to any vertex of $U$ then by connectivity and minimum degree condition of $G$ it is easy to find a vertex $u \in N(U)$ such that $u$ is an end vertex of a path of order 3. Hence Lemma 7 applies and gives a path of order $b$ using

$\left\lfloor\frac{b}{2}\right\rfloor-1$ vertices of $U$ which is a contradiction. Thus each vertex in $G-U$ is adjacent to a vertex of $U$.

Therefore $N(U)$ is exactly $G-U$. Lemma 7 implies that $G-U$ is $P_{3}$-free and together with Lemma 8 this implies that $G-U$ contains at most one edge. Moreover, if $a$ or $b$ is even, Lemma 7 implies that $G-U$ is $P_{2}$-free. Hence $G-U$ contains no edges if $a$ or $b$ is even and contains at most one edge if both $a$ and $b$ are odd. Therefore, $G$ is a subgraph of $G_{F}(n)$. This completes the proof of Lemma 6 .

Now we are ready to prove Theorem 2.

Proof of Theorem 2. We proceed by induction on $k$, the number of components of $F$. The base case, $k=2$, is given by Theorem 5 . Assume the theorem is true for $2 \leqslant \ell \leqslant k-1$. Pick $j$ such that $1 \leqslant j \leqslant k$ and $F^{\prime}=F-P^{j}$ is not $(k-1) \cdot P_{3}$. Let $G$ be an extremal graph for $F$. By Theorem 1 (Erdős-Gallai) $G$ must contain a $P^{j}$. Clearly $G-P^{j}$ is $F^{\prime}$-free, thus $e\left(G-P^{j}\right) \leqslant e\left(G_{F^{\prime}}\left(n-v_{j}\right)\right)$. Simple algebra and applying induction to $G-P^{j}$ gives $e(G)-e\left(G-P^{j}\right)-\left(\begin{array}{c}v_{j} \\ 2\end{array}\right) \geqslant\left\lfloor v_{j} / 2\right\rfloor n$ i.e. the number edges between $P^{j}$ and $G-P^{j}$ is at least $\left(\left\lfloor v_{j} / 2\right\rfloor-\frac{1}{2}\right) n$. Thus, by Lemma $4, P^{j}$ contains a set $U_{j}$ of $\left\lfloor v_{j} / 2\right\rfloor$ vertices who have at least $\sum_{i=1}^{k} v_{i}$ neighbors in $G-P^{j}$ (for $n$ large enough). Therefore $G-U_{j}$ must be $F^{\prime}$-free and by the induction hypothesis $G-U_{j}$ is $G_{F^{\prime}}$, so $G \subseteq G_{F}(n)$. Hence $G_{F}(n)$ is the unique extremal graph.

\section{$3 \quad$ Star forests}

In this section we give a proof of Theorem 3. Without going into all the details we remark that our proof gives that the size of $n$ for which the result holds depends on $\sum_{i=1}^{k} d_{i}$.

Let $F=\bigcup_{i=1}^{k} S^{i}$ be a star forest as in the statement of Theorem 3. Let $d_{i}$ be the maximum degree of $S^{i}$. Recall that $d_{1} \geqslant d_{2} \geqslant \cdots \geqslant d_{k}$.

We begin by describing the extremal graph for $F$. Let $F(n, i)$ be a graph obtained by adding a set $U$ of $i-1$ universal vertices to an extremal graph, $H$, for $S^{i}$ on $n-i+1$ vertices (see Figure 4$)$. Observe that $H$ is a $\left(d_{i}-1\right)$-regular graph if one of $\left(d_{i}-1\right)$ and $n-i+1$ is even and $n$ large enough (see [16]). If both are odd, then $H$ has exactly one vertex of degree $\left(d_{i}-2\right)$ and the remaining vertices have degree $\left(d_{i}-1\right)$. Therefore, we have $e(H)=\left\lfloor\frac{d_{i}-1}{2}(n-i+1)\right\rfloor$.

Observe that for all $1 \leqslant i \leqslant k, F(n, i)$ is $F$-free. Indeed, each star $S^{1}, \ldots, S^{i-1}$ must have at least one vertex from $U$ and $S^{i}$ is not a subgraph of $F(n, i)-U$.

Throughout the proof, unless otherwise specified, $i$ is always the index maximizing the number of edges. Notice that $e(F(n, i))$ is equal to the Turán number claimed by the theorem, i.e.

$$
e(F(n, i))=\left\lfloor\left(i-1+\frac{d_{i}-1}{2}\right) n-\frac{i-1}{2}\left(i+d_{i}-1\right)\right\rfloor .
$$



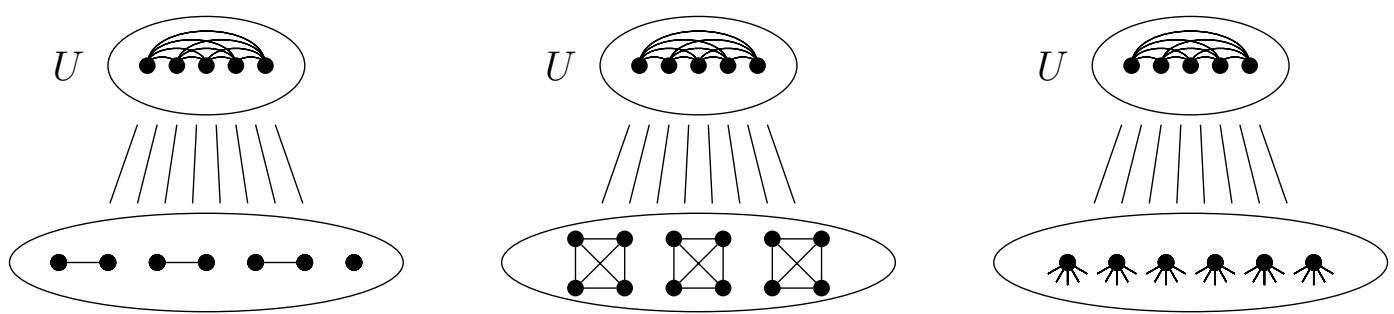

Figure 4: The extremal graph for a star-forest.

Define $f_{i}=i-1+\frac{d_{i}-1}{2}$, namely $f_{i}$ is the coefficient of $n$, the leading term of $e(F(n, i))$. We first show that for any $j<i, f_{j}<f_{i}$. Indeed, if $f_{j}>f_{i}$, then $j$ would be the index maximizing the number of edges, a contradiction. Thus we may assume $f_{j}=f_{i}$, namely by definition, $i-1+\frac{d_{i}-1}{2}=j-1+\frac{d_{j}-1}{2}$ and

$$
d_{j}=d_{i}+2(i-j)
$$

Thus we have:

$$
\begin{aligned}
e(F(n, j))-e(F(n, i)) & \geqslant \frac{i-1}{2}\left(i+d_{i}-1\right)-\frac{1}{2}-\frac{j-1}{2}\left(j+d_{j}-1\right) \\
& =\frac{i-1}{2}\left(i+d_{i}-1\right)-\frac{1}{2}-\frac{j-1}{2}\left(j+d_{i}+2(i-j)-1\right) \quad \text { by }(1) \\
& =\frac{i}{2}\left(d_{i}+i-1\right)-\frac{j}{2}\left(d_{i}+2 i-j-1\right)+\frac{1}{2}(i-j)-\frac{1}{2} \\
& =\frac{i}{2}\left(d_{i}+i\right)-\frac{j}{2}\left(d_{i}+i-j\right)-\frac{j i}{2}-\frac{1}{2} \\
& =\frac{i-j}{2}\left(d_{i}+i-j\right)-\frac{1}{2} \\
& >0
\end{aligned}
$$

But then $e(F(n, j))>e(F(n, i))$, contradicting the choice of $i$.

We use induction on $k$, the number of components of $F$. We prove the theorem in three cases distinguished by the index $i$ that maximizes the number of edges: (1) $i=k$, (2) $1<i<k$, and (3) $i=1$. Throughout this section we say a vertex has linear degree if the degree of the vertex is linear in $n$.

Case 1: $i=k$

Let $F^{\prime}=F-S^{k}$. Let $G$ be an extremal graph for $F$ with $n$ vertices. By the induction hypothesis, we have $\operatorname{ex}\left(n, F^{\prime}\right)=e\left(F\left(n, i^{\prime}\right)\right)$, where $i^{\prime}$ is the index maximizing the number of edges. Since $i=k$, we have $i^{\prime}<i$. Then as shown above, we have

$$
f_{i^{\prime}}<f_{i}=f_{k}
$$

Note that $F(n, i)$ is $F$-free, we have

$$
\operatorname{ex}\left(n, F^{\prime}\right)=f_{i^{\prime}} n+O\left(d_{i^{\prime}}\right)<f_{k} n+O\left(d_{k}\right)=e(F(n, k)) \leqslant e(G) .
$$


Thus $F^{\prime}=S^{1} \cup S^{2} \cup \cdots \cup S^{k-1} \subseteq G$ by the induction hypothesis.

It suffices to prove that there exists a vertex subset $U \subseteq V(G)$ of order $k-1$, such that every vertex in $U$ has linear degree, that is, $d(v)=\Omega(n)$. Indeed, if such a $U$ exists, then $G-U$ must be $S^{k}$-free. Otherwise, say there is a $S^{k}$ in $G-U$, then we can get $F^{\prime}$ using vertices in $U$ as centers and their neighbors in $G-U-S^{k}$ as leaves, which gives a copy of $F^{\prime} \cup S^{k}=F$, which is a contradiction. Hence $G \subseteq F(n, k)$ as desired.

Now we prove that such a $U$ exists. We know $F^{\prime} \subseteq G$, namely there are $k-1$ disjoint stars in $G$. Take any one of them, say $S^{j}, 1 \leqslant j \leqslant k-1$. Notice that $G-S^{j}$ has to be $F^{\prime}$-free, since otherwise a copy of $F^{\prime}$ in $G-S^{j}$ together with $S^{j}$ yields a copy of $S^{1} \cup S^{2} \cup \cdots \cup S^{k-1} \cup S^{j}$. Since $S^{k} \subseteq S^{j}$ we get that $F \subseteq G$, which is a contradiction. Note that $e\left(G\left[S^{j}\right]\right) \leqslant\left(\begin{array}{c}d_{j}+1 \\ 2\end{array}\right)$ and $e(G) \geqslant e(F(n, k))$. Let $e_{0}$ be the number of edges between $S^{j}$ and $G-S^{j}$. Then we have

$$
\begin{aligned}
e_{0} & =e(G)-e\left(G-S^{j}\right)-e\left(G\left[S^{j}\right]\right) \\
& \geqslant e(F(n, k))-\operatorname{ex}\left(n, F^{\prime}\right)-\left(\begin{array}{c}
d_{j}+1 \\
2
\end{array}\right) \\
& \sim f_{k} n-f_{i^{\prime}} n=\Omega(n)
\end{aligned}
$$

Thus there is a vertex in $S^{j}$ with linear degree. This is true for every $j$ with $1 \leqslant j \leqslant$ $k-1$, so consider a set containing a vertex of linear degree in each star. These $k-1$ vertices form the desired set $U$.

This finishes the proof of Case 1.

Case 2: $1<i<k$

Let $F^{*}=S^{i} \cup S^{i+1} \cup \cdots \cup S^{k}$ and $F^{\prime}=F-F^{*}$. As before, if $i^{\prime}$ is the index maximizing the number of edges for extremal graph of $F^{\prime}$, then $f_{i^{\prime}}<f_{i}$. A crucial observation about $F^{*}$ is the following.

$$
\operatorname{ex}\left(n, F^{*}\right)=\left\lfloor\frac{d_{i}-1}{2} n\right\rfloor .
$$

To show (3), notice that $i$ is the index maximizing the number of edges for $F$, thus for any $\ell>i, f_{\ell} \leqslant f_{i}$ or $\ell-1+\frac{d_{\ell}-1}{2} \leqslant i-1+\frac{d_{i}-1}{2}$. This implies

$$
\ell-i+\frac{d_{\ell}-1}{2} \leqslant \frac{d_{i}-1}{2} .
$$

By the induction hypothesis, if $i^{*}$ with $i \leqslant i^{*} \leqslant k$ is the index maximizing the size of the extremal graph for $F^{*}=S^{i} \cup S^{i+1} \cup \cdots \cup S^{k}$, we have,

$$
\begin{aligned}
\operatorname{ex}\left(n, F^{*}\right) & =\max _{i \leqslant \ell \leqslant k}\left\{\left[\ell-1+\frac{d_{\ell}-1}{2}\right] n-\left\lfloor\frac{\ell-1}{2}\left(\ell+d_{\ell}-1\right)\right\rfloor\right\} \\
& =\left[i^{*}-1+\frac{d_{i^{*}}-1}{2}\right] n-\left\lfloor\frac{i^{*}-1}{2}\left(i^{*}+d_{i^{*}}-1\right)\right\rfloor \\
& \leqslant\left[i^{*}-1+\frac{d_{i^{*}}-1}{2}\right] n \\
& \leqslant \frac{d_{i}-1}{2} n
\end{aligned}
$$$$
\text { by (4) and } i^{*} \geqslant i \text {. }
$$ 
On the other hand, a $S^{i}$-free graph is $F^{*}$-free, thus $\operatorname{ex}\left(n, F^{*}\right) \geqslant\left\lfloor\frac{d_{i}-1}{2} n\right\rfloor$.

Let $G$ be an extremal graph for $F$ on $n$ vertices. Recall $F^{\prime}=S^{1} \cup \cdots \cup S^{i-1}$, the choice of $i$ and the extremality of $G$ implies $e(G) \geqslant e(F(n, i))>\operatorname{ex}\left(n, F^{\prime}\right)$. Thus $F^{\prime} \subseteq G$. As before if we can show there is a vertex with linear degree in each star in $F^{\prime} \subseteq G$, then we have a set $U$ of order $i-1$, each vertex of which has linear degree and $G-U$ is $F^{*}$-free. Then by (3) we get $G \subseteq F(n, i)$.

Take any star in a copy of $F^{\prime}$ in $G$, say $S^{j}$, note that $j<i$. We take the same approach as before to prove that the number of edges between $S^{j}$ and $G-S^{j}$ is linear, namely $e_{0}=\Omega(n)$, which implies the existence of a vertex with linear degree as desired.

Note that $G-S^{j}$ must be $\left(F-S^{j}\right)$-free. We now give an upper bound on $e\left(G-S^{j}\right)$. Let $F^{\prime \prime}=F-S^{j}$ and let $i^{\prime \prime}$ be the index maximizing the number of edges for $F^{\prime \prime}$.

If $i^{\prime \prime}<j<i$, then $f_{i^{\prime \prime}}<f_{i}$ and thus

$$
e\left(G-S^{j}\right) \leqslant \operatorname{ex}\left(n, F^{\prime \prime}\right) \leqslant f_{i^{\prime \prime}} n .
$$

Therefore, $e_{0}=e(G)-e\left(G\left[S^{j}\right]\right)-e\left(G-S^{j}\right) \geqslant f_{i} n-f_{i^{\prime \prime}} n-\left(\begin{array}{c}d_{j}+1 \\ 2\end{array}\right)=\Omega(n)$ as desired.

Notice that in $F^{\prime \prime}$, all indices after $j$ were shifted to the left by one, that is $F^{\prime \prime}=$ $S^{1} \cup \cdots \cup S^{j-1} \cup S^{j+1} \cup \cdots \cup S^{k}$. Thus if $i^{\prime \prime} \geqslant j$, by the definition of $f_{i}$, then it is the same index as $F$ that maximizes the number of edges for $F^{\prime \prime}$, that is $i$ in $F$ and $i-1$ in $F^{\prime \prime}$.

Thus $i^{\prime \prime}=i-1$. In this case $e\left(G-S^{j}\right) \leqslant[(i-1)-1]+\frac{d_{i}-1}{2}=f_{i}-1$. Thus $e_{0} \geqslant f_{i} n-f_{i^{\prime \prime}} n-\left(\begin{array}{c}d_{j}+1 \\ 2\end{array}\right)=n-\left(\begin{array}{c}d_{j}+1 \\ 2\end{array}\right)=\Omega(n)$ as desired.

This finishes the proof of Case 2 .

Case 3: $i=1$.

Let $G$ an extremal graph for $F$. We want to show that $e(G)=\operatorname{ex}(n, F)=\left\lfloor\frac{d_{1}-1}{2} n\right\rfloor$. Since an $S^{1}$-free graph is $F$-free, $e(G) \geqslant\left\lfloor\frac{d_{1}-1}{2} n\right\rfloor$.

We may assume $\Delta(G) \geqslant d_{1}$, since otherwise $e(G) \leqslant \frac{d_{1}-1}{2} n$. Let $v$ be a vertex of degree $\Delta(G)$, so $d(v) \geqslant d_{1}$. Thus we can get a $S^{1}$ from $N(v) \cup\{v\}$ with $v$ as its center. Note that since $i=1$, we have for any $j>1, f_{j} \leqslant \frac{d_{1}-1}{2}$. Let $F^{*}=F-S^{1}=S^{2} \cup \cdots \cup S^{k}$. Observe that in $F^{*}$, all indices were shifted to the left by one. Hence if $i^{*}$ is the index maximizing the number of edges for $F^{*}$, then it was $j=i^{*}+1 \geqslant 2$ in $F$. Thus $f_{i^{*}}=$ $[(j-1)-1]+\frac{d_{j}-1}{2}=f_{j}-1 \leqslant \frac{d_{1}-1}{2}-1$.

Let $e_{0}$ be the number of edges between $S^{1}$ and $G-S^{1}$, then $e_{0}=e(G)-e\left(\left[S^{1}\right]\right)-$ $e\left(G-S^{1}\right) \geqslant \frac{d_{1}-1}{2} n-\left(\begin{array}{c}d_{1}+1 \\ 2\end{array}\right)-f_{i^{*}} n \geqslant n-\left(\begin{array}{c}d_{1}+1 \\ 2\end{array}\right)=\Omega(n)$. Thus there is a vertex of linear degree in $S^{1}$, let it be $u$. This implies $G-\{u\}$ is $F^{*}$-free. Thus

$$
e(G)=d(u)+e(G-u) \leqslant n-1+\operatorname{ex}\left(n, F^{*}\right) \leqslant n-1+f_{i^{*}} n \leqslant \frac{d_{1}-1}{2} n-1<e(F(n, 1)),
$$

which is a contradiction.

This finishes the proof of Case 3 and therefore Theorem 3.

\section{Forests with components of order 4}

Now we consider forests with components each of order 4 . Notice that there are only two trees of order 4: the path $P_{4}$ and the star $S_{3}$. Let $F=a \cdot P_{4} \cup b \cdot S_{3}$. Let $G_{F}^{1}(n)$ be the 
$n$-vertex graph constructed as follows: assume $n-b=3 d+r$ with $r \leqslant 2, G_{F}^{1}(n)$ contains $b$ universal vertices, the remaining graph is $K_{r} \cup d \cdot K_{3}$. Let $G_{F}^{2}(n)$ be the $n$-vertex graph containing $2 a+b-1$ universal vertices and the remaining graph is empty. See Figure 5 . It is easy to check that both $G_{F}^{1}(n)$ and $G_{F}^{2}(n)$ are $F$-free as the set of universal vertices is too small to accommodate all components of $F$.
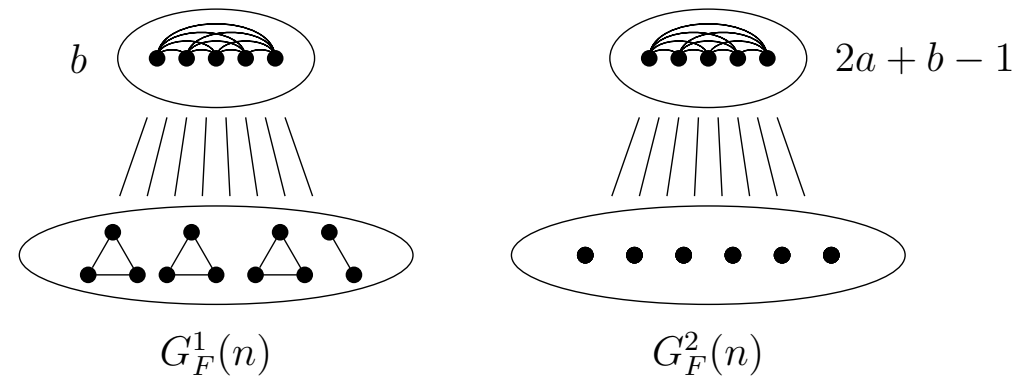

Figure 5: The extremal graphs for a forest with components of order 4 .

Theorem 9. Let $F=a \cdot P_{4} \cup b \cdot S_{3}$, and $n=3 d+r$ be sufficiently large with $r \leqslant 2$, then

(i) If $a=1$ and $r=0$, then $G_{F}^{1}(n)$ is the unique extremal graph; if $a=1$ and $r \neq 0$, then $G_{F}^{1}(n)$ and $G_{F}^{2}(n)$ are the only extremal graphs for $F$.

(ii) If $a>1$, then $G_{F}^{2}(n)$ is the unique extremal graph for $F$.

We only give a sketch of the proof. Use induction on $b$, the number of copies of $S_{3}$. Let $G$ be an extremal graph with $n$ vertices, then $e(G) \geqslant e\left(G_{F}^{i}(n)\right), i=1,2$. Thus $G$ contains a copy of $S_{3}$, similar as the proof for star-forest, any copy of $S_{3}$ in $G$ contains a vertex, say $v$, of degree $\Omega(n)$. Then $G-\{v\}$ must be $F^{\prime}$-free where $F^{\prime}=a \cdot P_{4} \cup(b-1) \cdot S_{3}$. Then by the inductive hypothesis, $G-\{v\}$ has to be $G_{F^{\prime}}^{i}(n-1)$, which then implies $G \subseteq G_{F}^{i}(n)$. The base case of the induction is when $b=0$, then the graph is $a \cdot P_{4}$. This also explains why we have two different constructions for the extremal graphs. Because when $a=1$, by a result of Faudree and Schelp [10], vertex-disjoint copies of triangles or a star are the extremal graphs (actually, they showed a combination of triangles and a smaller star is also an extremal case, but here, if any triangle appears, then the number of universal vertices will be fewer which yields a construction worse than $G_{F}^{1}$ and $G_{F}^{2}$ ); when $a>1$, then Theorem 2 implies that $G_{F}^{2}(n)$ is the unique extremal graph.

The same technique can be applied to $a \cdot P_{\ell} \cup b \cdot S_{t}$, but the proof is very technical.

\section{Acknowledgments}

We would like to thank József Balogh, Alexandr Kostochka and Douglas B. West for encouragement and fruitful discussions and the anonymous referee for suggested improvements of the presentation of the results. 


\section{References}

[1] B. Bollobás. Modern Graph Theory. Springer Science+Business Meda, Inc., USA, 3rd edition, 1998.

[2] J. A. Bondy and M. Simonovits. Cycles of even length in graphs. J. Combinatorial Theory Ser. B, 16:97-105, 1974.

[3] S. Brandt. Subtrees and subforests of graphs. J. Comb. Theory, Ser. B, 61(1):63-70, 1994.

[4] W. G. Brown. On graphs that do no contain a Thomsen graph. Canad. Math. Bull., 9:281-289, 1966.

[5] N. Bushaw and N. Kettle. Turán numbers of multiple paths and equibipartite forests. Combinatorics, Probability and Computing, 20:837-853, 2011.

[6] P. Erdős. Extremal problems in graph theory. Theory of Graphs and its Applications (Proc. Sympos. Smolenice, 1963), pages 29-36, 1964.

[7] P. Erdős and T. Gallai. On maximal paths and circuits of graphs. Acta Math. Acad. Sci. Hungar., 10:337-356, 1959.

[8] P. Erdős, A. Rényi, and V. T. Sós. On a problem of graph theory. Studia Sci. Math. Hungar., 1:215-235, 1966.

[9] P. Erdős and A. H. Stone. On the structure of linear graphs. Bull. Amer. Math. Soc., 52:1087-1091, 1946.

[10] R. J. Faudree and R. H. Schelp. Path Ramsey numbers in multicolorings. J. Combinatorial Theory Ser. B, 19:150-160, 1975.

[11] Z. Füredi. New asymptotics for bipartite Turán numbers. J. Combin. Theory Ser. A, 75:141-144, 1996.

[12] Z. Füredi, T. Jiang, and R. Seiver. Exact solution of the hypergraph Turán problem for $k$-uniform linear paths, 2011. submitted. arXiv:1108.1247

[13] M. K. Goldberg and M. Magdon-Ismail. Embedding a forest in a graph. Electr. J. Comb., 18(1):\#P99, 2011.

[14] I. Gorgol. Turán numbers for disjoint copies of graphs. Graphs and Combinatorics, 27(5):661-667, 2011.

[15] T. Kővári, V. T. Sós, and P. Turán. On a problem of K. Zarankiewicz. Colloquium Math., 3:50-57, 1954.

[16] M. Simonovits. A method for solving extremal problems in graph theory, stability problems. Theory of Graphs (Proc. Colloq., Tihany, 1966), pages 279-319, 1968.

[17] P. Turán. Egy gráfelméleti szélsőértékfeladatról. Mat. es Fiz. Lapok, 48:436-452, 1941.

[18] P. Turán. On the theory of graphs. Colloquium Math, 3:19-30, 1954. 